\title{
Prevalencia del consumo de cigarrillos y otras formas de tabaco en estudiantes universitarios
}

Fabio Bautista-Pérez ${ }^{1}$ Recibido: 20/02/14 Recibido: 19/06/14

\section{Resumen}

El objetivo del presente estudio fue determinar la prevalencia del consumo de cigarrillos u otra forma de tabaco en estudiantes de la Universidad Evangélica de El Salvador, San Salvador, El Salvador. Estudio descriptivo con un diseño transversal y una muestra de 430 estudiantes, con un promedio de edad de 21,72 años y una DS de 3,7. Se realizó un análisis descriptivo de la información y se aplicó la prueba Chi-cuadrada. La distribución de la muestra por sexo es de 33,3\% hombres y $66,7 \%$ mujeres. El $34,8 \%$ había fumado cigarrillo alguna vez en la vida, $11,8 \%$ en los últimos 12 meses y 9,4\% en el último mes; al relacionarlo con el sexo resultaron estadísticamente significativos $\left(\chi^{2}=219,44, P \approx 0,000\right)$. Al comparar edad con consumo de cigarrillos se observó que en las edades de 19 a 24 años se presentaron los consumos mayores para una vez en la vida, 12 meses y 30 días, predominando la edad de 23 a 24 años con los mayores porcentajes. Se encontraron diferencias estadísticas significativas para una vez en la vida $\left(\chi^{2}=182,49, P \approx 0,000\right)$; últimos 12 meses $\left(\chi^{2}=111,24, P \approx 0,000\right)$ y últimos 30 días $\left(\chi^{2}=87,74, P \approx 0,000\right)$. La edad promedio de inicio de consumo de cigarrillo, fue de 15,60 años. La prevalencia de consumo de cigarrillos u otras formas de tabaco entre los estudiantes universitarios resultó elevada, lo cual indica que es necesario realizar estudios de prevención para reducir el consumo y desarrollar programas de abandono del tabaquismo para consumidores definidos.

Palabras clave: Prevalencia, consumo de cigarrillos, tabaquismo, estudiante universitario, El Salvador. 


\section{Introducción}

El tabaquismo es una enfermedad adictiva crónica, una "pandemia" que afecta a todos los grupos étnicos y no tiene fronteras ni limitaciones de género, edad, estrato sociocultural o religión. En la actualidad, el consumo de tabaco es uno de los principales problemas de salud pública en los países desarrollados y comienza a serlo en los países en vías de desarrollo, no sólo por su magnitud en términos del número de sujetos afectados, sino por las enormes consecuencias sociales, económicas y sanitarias que conlleva. ${ }^{1-3}$

La Comisión Nacional Antidrogas (CNA), ${ }^{4}$ evidenció que el consumo de cigarrillos una vez en la vida fue de $27,9 \%$, donde el $34,3 \%$ corresponde al género masculino y $22,4 \%$ al femenino. En los últimos doce meses, la prevalencia de consumo fue $15,1 \%$, el género masculino presentó $19,9 \%$ y el femenino 10,9\%; en los últimos 30 días el consumo fue de $9,3 \%$, el género masculino presentó $13,2 \%$ y el femenino 5,9\%. ${ }^{5,6}$

En Chile, ${ }^{7}$ se encontró un $18,6 \%$ de consumo de cigarrillos; en México ${ }^{8}$ fue de 9,7\% (IC 95\%: 8,9 a 10,6) entre los adolescentes que declararon que habían fumado por lo menos cien cigarrillos de tabaco durante toda su vida; en una universidad de Colombia, ${ }^{9}$ reportan la prevalencia de fumadores en estudiantes con un $31 \%$ de casos, seguido por el grupo de administrativos con el $20,17 \%$ y el grupo de profesores con el $17 \%$ de fumadores. La edad estaba entre los 22 y los 45 años. En Venezuela, ${ }^{10}$ se determinó que la edad promedio de inicio en el consumo fue de $14,99 \pm 2,67$ años. Se evidenció que $27,4 \%$ de los estudiantes encuestados ha consumido tabaco alguna vez en la vida. En relación al tipo de tabaco consumido por los estudiantes de nuevo ingreso a la
Facultad de Ciencias de la Salud, se encontró que $24,1 \%$ del total consume cigarrillos. El $3,9 \%$, consume tabaco (habanos o puros), $3,6 \%$ fuma en pipa y llama la atención que $3,4 \%$, consume chimó, forma de tabaco que ha sido fabricado artesanalmente en Venezuela (es una jalea de tabaco).

En universidades de España, ${ }^{11,12}$ encontraron una prevalencia de fumadores del $30,3 \%$. En una universidad Peruana, ${ }_{1}^{13}$ se encontró que el $79,9 \%$ de la población estudiada refiere haber fumado alguna vez en su vida. La edad de inicio del tabaquismo, tiene un rango de 9 a 25 años con una media de 15,54 años y una $D E \pm 2,11$, con el 51,09 $\%$. La prevalencia del tabaquismo es mayor en el sexo masculino con el $84,64 \%$ frente a la prevalencia en el sexo femenino con el $76,03 \%$. El $55.94 \%$ de estudiantes fuma entre uno a cinco cigarrillos diarios. Sólo el $0,56 \%$ de los estudiantes fuma más de media cajetilla diaria. El $18,06 \%$ del total de encuestados era portador de síntomas sugestivos de asma.

En estudio de una universidad de Argentina, ${ }^{14}$ en 400 alumnos, encontraron una prevalencia de fumadores actuales (Habituales + Ocasionales) de 31,75\% (127) de los cuales $22,25 \%$ (93) son habituales y un $9,5 \%$ (34) ocasionales. Observaron que el $47 \%$ fuma menos de 10 cigarrillo/día, el $41 \%$ entre 10-20 cigarrillos/día y el $12 \%$ restante más de 20 cigarrillos/día. El 100\% de los encuestados admitieron que el fumar resulta perjudicial para la salud. El 59,7\% (163) de los no fumadores se considera fumador pasivo y un $93 \%$ de los no fumadores lo consideran muy perjudicial para su salud.

El presente estudio tiene como propósito determinar la prevalencia del consumo de cigarrillos y otras formas de consumo de tabaco en estudiantes universitarios. Esto servirá de base para desarrollar programas de 
prevención y/o tratamiento a los estudiantes que ya sufren adicción al consumo de tabaco, para que reduzcan el consumo o dejen de fumar en forma voluntaria.

El enunciado del problema de estudio fue: ¿Es posible que estudiantes consuman cigarrillos y otras formas de tabaco en una universidad privada de El Salvador durante el año 2013?

\section{Metodología de la investigación}

\section{Ubicación}

El estudio se realizó en una universidad privada de El Salvador durante el año 2013, donde se aplicó un cuestionario sobre consumo de cigarrillos $u$ otras formas de tabaco a los estudiantes de pregrado de las seis facultades que posee la universidad. El estudio fue de tipo descriptivo con un diseño transversal.

\section{Población y muestra}

La población del estudio fue de 3,700 estudiantes de pregrado que estaban matriculados en la universidad. La muestra calculada fue de 430 estudiantes; esta fue determinada utilizando el programa de internet Survey System Calculator. Este programa está basado en los intervalos de confianza al $95 \%$ de significancia ${ }^{15}$.

\section{Criterios de inclusión}

Los estudiantes que participaron en el estudio estaban matriculados en la Universidad, lo que fue comprobado mediante su carné de identificación. El estudio fue sometido a la aprobación del Comité de Ética para la Investigación de la Universidad Evangélica de El Salvador. Se aseguró a los estudiantes que la información proporcionada era confidencial y anónima para salvaguardar su identidad. La participación de los estudiantes fue voluntaria y tenían la libertad de no continuar participando en el momento que ellos decidieran por alguna razón que ellos consideren lesiva a su persona.

\section{Instrumento}

En el estudio se utilizó un instrumento de 47 preguntas, donde 22 eran de carácter sociodemográfico y el resto para indagar características relacionadas con el consumo de cigarrillos u otras formas de consumo de tabaco. En la elaboración del instrumento se utilizaron como base preguntas de un instrumento ya existente sobre consumo de drogas. ${ }^{16}$

\section{Recolección de la información}

El proceso de recolección de la información del estudio fue conducido por el investigador principal y colaboradores entrenados para tal fin. Este proceso se realizó después de la presentación y aprobación del proyecto de estudio ante las respectivas autoridades y organismos al interior de la universidad [Consejo de Investigación e innovación institucional (COIN), Dirección de Investigación (DI), Vicerrectoría de Investigación y Proyección Social (VRIPS) y ante el Comité de Ética en Investigación (CEI)]. El cuestionario fue completado por los estudiantes en aproximadamente 20 minutos.

\section{Elaboración de la base de datos}

La base de datos y el análisis de la información se realizó en el "Programa Estadístico para las Ciencias Sociales" (SPSS, siglas en Inglés), Versión 14.0 para Windows. ${ }^{17}$ El ingreso y análisis de los datos fue realizado por el investigador principal del proyecto y un asistente de investigación. La base de datos original fue corregida adecuadamente para evitar inconsistencias, datos perdidos $y$ datos incorrectos. 


\section{Análisis de los datos}

La información obtenida fue analizada descriptivamente, determinando frecuencias, intervalos de confianza, relaciones de género y grupos de edades con respecto a las variables en estudio. A estas relaciones se les aplicó la prueba de independencia Chi Cuadrado y un análisis de riesgo en el consumo de cigarrillos y otras formas de tabaco a través de un análisis de regresión logística, en ambas pruebas se consideró un nivel de significancia al 0,05.

\section{Análisis de resultados y discusión}

\section{Información sociodemográfica}

La población del estudio fue de 3700 estudiantes y la muestra estaba constituida por 430 , con un promedio de edad de 21,72 años y una desviación estándar (D.E.) de 3,7, se encontró que el $33,3 \%$ correspondió al género masculino y $66,7 \%$ al femenino.

\section{Prevalencia del consumo de tabaco}

En general, el 34,8\% mencionó haber fumado cigarrillo alguna vez en la vida, $11,8 \%$ en los últimos 12 meses y $9,4 \% \%$ en el último mes (Figura 1).

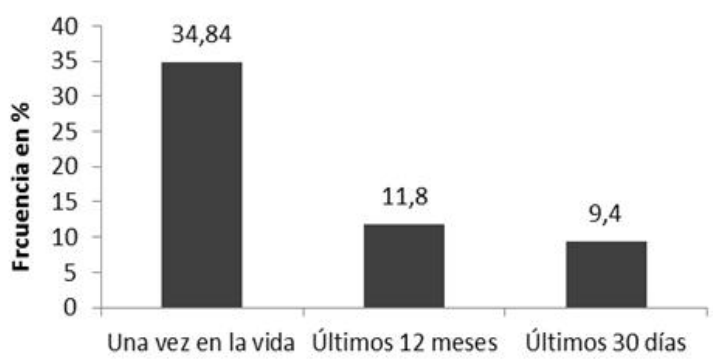

Figura 1. Prevalencia del consumo de tabaco

Al relacionar género con consumo de cigarrillos se encontró que el porcentaje de consumo para el género masculino para una vez en la vida y en los últimos 12 meses fue el doble en relación al género femenino, resultando estadísticamente significativos en ambos casos $\left(\chi^{2}=219,44, \mathrm{P} \approx 0,000 ; \chi 2=80,86\right.$, $p \approx 0,000$ ). Sin embargo, en el consumo para el último mes se observó que el género femenino presentó un consumo más de tres veces en relación al género masculino, resultando estadísticamente significativo $\left(\left(\chi^{2}=74,79, P \approx 0,000\right)\right.$ (Figura 2).

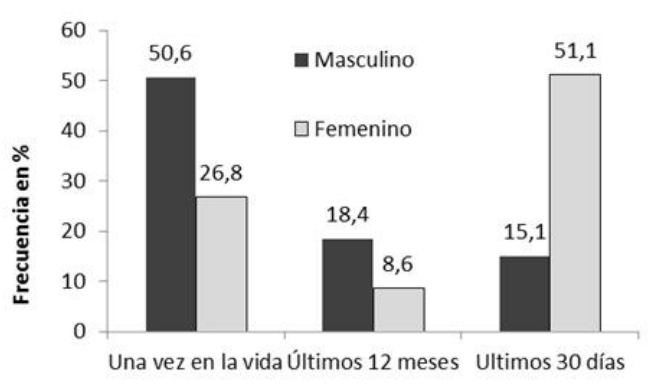

Figura 2. Distribución del consumo de tabaco por género

Al comparar edad con consumo de cigarrillos se observó que en las edades de 19 a 24 años se presentaron los consumos mayores para una vez en la vida, 12 meses y 30 días, predominando la edad de 23 a 24 años con los mayores porcentajes. Los menores porcentajes los mostraron las edades de 16 a 18 años y 25 o más años, en los tres casos se observó la tendencia a incrementar el consumo a medida se incrementaba la edad hasta los 24 años, luego disminuía (Tabla 1). Se encontraron diferencias estadísticas significativas para una vez en la vida $\left(\chi^{2}=182,49, P \approx 0,000\right)$; últimos 12 meses $\left(\chi^{2}=111,24, \quad P \approx 0,000\right)$ y últimos 30 días $\left(\chi^{2}=87,74, P \approx 0,000\right)$.

Tabla 1. Distribución de consumo de cigarrillos edad

\begin{tabular}{lccc}
\hline Edad & $\begin{array}{c}\text { Una vez en } \\
\text { la vida* }\end{array}$ & $\mathbf{1 2}$ meses* & 30 días * \\
\hline 16-18 años & $19,2 \%$ & $2,3 \%$ & $2,0 \%$ \\
$19-20$ años & $35,4 \%$ & $10,0 \%$ & $8,3 \%$ \\
$21-22$ años & $34,0 \%$ & $15,1 \%$ & $11,2 \%$ \\
23-24 años & $47,3 \%$ & $20,3 \%$ & $16,1 \%$ \\
25 o más años & $32,0 \%$ & $7,3 \%$ & $5,8 \%$ \\
Total & $34,8 \%$ & $11,8 \%$ & $9,4 \%$ \\
${ }^{*}$ P<0,05 & & & \\
\hline
\end{tabular}


Una característica estudiada fue la edad de inicio de consumo de cigarrillo, observando que la edad promedio fue de 15,60 años, donde el género masculino mostró un inicio más temprano con relación al femenino. También se obtuvo que en los últimos 30 días los estudiantes mencionaron que habían fumado cigarrillos u otro tipo de tabaco un promedio de 2,29 días.

El consumo de cigarrillos de acuerdo a la "situación económica del estudiante" mostró que $34,8 \%$ lo hizo una vez en la vida, $11,8 \%$ lo hizo en los últimos 12 meses y $9,4 \%$ en los últimos 30 días. Al comparar las variables "situación económica" con "consumo de cigarrillos", se observó que la situación económica "Buena y Regular", presentaron los porcentajes mayores de consumo de cigarrillos. La situación económica "muy mala", no presentó consumo para una vez en la vida y últimos 12 meses, pero se observó un consumo elevado para los últimos 30 días (Tabla 2). Para estos contrastes se encontraron diferencias estadísticas significativas para una vez en la vida $\left(\chi^{2}=76,67, P \approx 0,000\right)$, últimos 12 meses $\left(\chi^{2}=126,16, P \approx 0,000\right)$ y últimos 30 días $\left(\chi^{2}=184,25, P \approx 0,000\right)$.

Tabla 2. Distribución del consumo de cigarrillo de acuerdo a la situación económica

\begin{tabular}{lccc}
\hline $\begin{array}{l}\text { Situación } \\
\text { económica }\end{array}$ & Vida* & $\mathbf{1 2}$ meses* $^{*}$ & 30 días* \\
\hline Muy buena & $22,8 \%$ & $5,6 \%$ & $4,4 \%$ \\
Buena & $38,4 \%$ & $15,6 \%$ & $13,3 \%$ \\
Regular & $35,2 \%$ & $7,7 \%$ & $6,2 \%$ \\
Mala & $27,8 \%$ & $20,7 \%$ & $2,2 \%$ \\
Muy Mala & $0,0 \%$ & $0,0 \%$ & $55,6 \%$ \\
Total & $34,8 \%$ & $11,8 \%$ & $9,4 \%$ \\
${ }^{*} \mathrm{p}<0,05$ & & & \\
\hline
\end{tabular}

El consumo de cigarrillos entre familiares mostró que $25,8 \%$ poseía familiares que fumaban, observando que los hombres poseían más familiares que fumaban que entre las mujeres, que resultó menor, esta relación fue estadísticamente significativa $\chi 2=54,56, P \approx 0,000$ ) (Tabla 3).

Tabla 3. Consumo de cigarrillos entre familiares con relación al género.*

\begin{tabular}{lcc}
\hline Género & Si & No \\
\hline Masculino & $30,0 \%$ & $56,8 \%$ \\
Femenino & $23,7 \%$ & $54,2 \%$ \\
Total & $25,8 \%$ & $55,1 \%$ \\
${ }^{*} \mathrm{P}<0,05$ & & \\
\hline
\end{tabular}

Al contrastar la variable fumar es dañino para la salud con la edad se observó que a medida que aumenta la edad incrementa el porcentaje que afirma que fumar es dañino para la salud (Tabla 4). Este contraste resultó estadísticamente significativo $\left(\chi^{2}=108,04\right.$, $\mathrm{P} \approx 0,000$ ).

Tabla 4. Fumar es dañino para la salud contrastada con la edad.*

\begin{tabular}{lcc}
\hline Edad & $\mathbf{S i}$ & No/No Sabe \\
\hline $16-18$ años & $88,8 \%$ & $0,0 \%$ \\
$19-20$ años & $93,6 \%$ & $2,0 \%$ \\
$21-22$ años & $91,2 \%$ & $0,0 \%$ \\
$23-24$ años & $94,0 \%$ & $3,3 \%$ \\
25 o más años & $96,2 \%$ & $0,9 \%$ \\
Total & $92,9 \%$ & $1,3 \%$ \\
${ }^{*} P<0,05$ & & \\
\hline
\end{tabular}

En análisis de regresión logística realizado se encontró que las variables que resultaron predictoras de riesgo de consumo de tabaco fueron: Trabaja y estudia, Lugar donde compra los cigarrillos, Formas de consumo de tabaco, Observación o escucha de propaganda sobre consumo de cigarrillos y Fumar hace bajar de peso, las que pueden ser observadas en la Tabla 5. A partir de estos resultados se realizó el análisis de riesgo: "si fuma o no cigarrillos una vez en la vida". 
Tabla 5. Resultados de la regresión logística

\begin{tabular}{|c|c|c|c|c|c|}
\hline & & & & IC $95 \%$ para $\mathrm{E}$ & \\
\hline Variables & $\beta$ & p-valor & Exponente $\beta$ & Límite inferior & Límite superior \\
\hline Trabaja y estudia & & 0,000 & & & \\
\hline Si trabaja & $-0,459$ & 0,001 & 0,632 & 0,477 & 0,837 \\
\hline Lugar donde compra los cigarrillos & & 0,000 & & & \\
\hline Tienda & $-3,751$ & 0,000 & 0,023 & 0,007 & 0,075 \\
\hline Supermercado & $-3,846$ & 0,000 & 0,021 & 0,005 & 0,089 \\
\hline Kiosko & $-21,958$ & 0,999 & 0,000 & 0,000 & 0,000 \\
\hline Venta ambulante & -21.958 & 0.999 & 0.000 & 0,000 & 0,000 \\
\hline Bares & $-21,958$ & 0,999 & 0,000 & 0.000 & 0.000 \\
\hline Discotecas & -21.958 & 0.999 & 0.000 & 0.000 & 0.000 \\
\hline Formas de consumo de tabaco & & 0,000 & & & \\
\hline Cigarrillo & $-4,354$ & 0,000 & 0,013 & 0,006 & 0,028 \\
\hline Puro o habano & $-22,210$ & 0,999 & 0,000 & 0,000 & 0,000 \\
\hline Pipa & $-22,210$ & 0,999 & 0,000 & 0,000 & 0,000 \\
\hline $\begin{array}{l}\text { Observación o escucha de propaganda sobre } \\
\text { consumo de cigarrillos }\end{array}$ & & 0,53 & & & \\
\hline Mucha & $-0,344$ & 0,035 & 0,709 & 0,515 & 0,976 \\
\hline Poca & $-0,062$ & 0,684 & 0,940 & 0,696 & 1,268 \\
\hline Fumar hace bajar de peso & & 0,002 & & & \\
\hline $\mathrm{Si}$ & $-0,019$ & 0,906 & 0,981 & 0,718 & 1,342 \\
\hline No & $-0,434$ & 0,003 & 0,648 & 0,486 & 0,863 \\
\hline
\end{tabular}

\section{Discusión}

El consumo de cigarrillos por los estudiantes fue alto, más en los hombres que entre las mujeres, concordando con estudios anteriores que mencionan este tipo de situación ${ }^{9-14}$. Con respecto a la edad de consumo, se observó que desde los 16 años hasta los 24 años el consumo de cigarrillos aumentó y a partir de los 25 disminuyó, tanto para una vez en la vida, como para los últimos 12 meses y 30 días, lo que está de acuerdo con algunos autores que encontraron esta misma situación. ${ }^{17}$

Se observó que el consumo de cigarrillos en los estudiantes con la situación económica "Buena y Regular", presentaron los porcentajes mayores de consumo, para una vez en la vida y últimos 12 meses, esto posiblemente por tener una mayor capacidad de compra a través del tiempo, aunque para los últimos 30 días, se observó en los de situación económica muy mala presentaron los mayores porcentajes de consumo.

La edad promedio de inicio de consumo de cigarrillo, fue de 15,60 años, donde el género masculino mostró un inicio más temprano con relación al femenino, esto concuerda con otros estudios que mencionan que la edad de inicio es similar al encontrado en este estudio. $9,10,12,14$

El consumo de cigarrillos entre familiares mostró que $25,8 \%$ poseía familiares que fumaban, observando que los hombres poseías más familiares que fumaban que entre las mujeres, esto está de acuerdo a estudios que mencionan que encontraron hasta un $15 \%$ que viven con padres, familiares o personas que fuman (incluyendo también hijos y esposos). ${ }^{9}$ 
El 92,9\% de los estudiantes consideró que fumar es dañino para la salud, así como también el humo de cigarrillo, donde las mujeres presentaron el mayor porcentaje; también se observó que a medida que aumenta la edad incrementa el porcentaje que afirma que fumar es dañino para la salud, esto está de acuerdo a otros estudios donde mencionan que el $100 \%$ de los encuestados admiten que el fumar resulta perjudicial para la salud y un $93 \%$ de los no fumadores lo consideran muy perjudicial para su salud. ${ }^{14}$

\section{Conclusiones}

De acuerdo a los resultados obtenidos $y$ en las condiciones en que se realizó el estudio se concluye:

El consumo de cigarrillos por los estudiantes fue alto, más en los hombres que entre las mujeres. Se observó que el consumo se incrementó al aumentar la edad, llegando al punto más alto a los 24 años, después disminuyó tanto para una vez en la vida, en los últimos 12 meses y últimos 30 días.

La edad promedio de inicio de consumo de cigarrillo, fue de 15,60 años, donde el género masculino mostró un inicio más temprano con relación al femenino. Al relacionar consumo de cigarrillos por primera vez con los últimos 30 días, más de un mes y más de un año, con edad, se encontró que el porcentaje se incrementó a medida que aumentó la edad.

El consumo de cigarrillos está de acuerdo a la "situación económica del estudiante" a mejor situación económica más consumo en relación a los de situación más precaria, aunque estos fuman pero en menor proporción. Un porcentaje alto de los que fuman poseen familiares fumadores, siendo los hombres los que tienen el mayor porcentaje. También se observó que a medida que aumenta la edad incrementa el porcentaje que afirma que fumar es dañino para la salud.

\section{Recomendaciones}

Es necesario que en la Universidad se desarrollen programas de prevención del consumo de cigarrillos y otras formas de consumo de tabaco para reducir el tabaquismo entre los estudiantes.

Que la Universidad apoye el desarrollo y ejecución de programas de abandono o cesación del tabaquismo entre los estudiantes que ya fuman, lo cual va en beneficio del estudiante y del país.

Continuar los estudios de prevalencia del consumo de cigarrillos y otras formas de tabaco entre los estudiantes en forma bianual para medir cual es la tendencia del tabaquismo en la Universidad.

Que la Universidad apoye la creación de una clínica para el tratamiento del tabaquismo para los estudiantes que voluntariamente quieran abandonarlo.

\section{Agradecimientos}

El investigador agradece a las autoridades de la Universidad Evangélica de El Salvador por el apoyo recibido para realizar esta investigación. Asimismo se agradece a la Señorita Carmen Manzanares por participar en las diferentes actividades del estudio, a las autoridades de las facultades que permitieron la aplicación de la encuesta y a los estudiantes que voluntariamente contestaron el cuestionario.

\section{Fuentes consultadas}

1. Jiménez-Muro FA, San Agustín, AB, Marqueta Baile A, Gargallo Valero P, Nerín de la Puerta I. Consumo de drogas en estudiantes universitarios de primer curso. (En línea). Departamento de Medicina y Psiquiatría. Unidad de Tabaquismo 
FMZ. Facultad de Medicina, Universidad de Zaragoza. Departamento de Métodos Estadísticos. Escuela Universitaria de Estudios Empresariales, Universidad de Zaragoza. España. [Consultado noviembre 2012]. Disponible en: http://redalyc.uaemex.mx/src/inicio/ArtPdfRed. jsp?iCve $=289122882004$

2. Montes A, Pérez M, Gestal JJ. Impacto del tabaquismo sobre la mortalidad en España. (En línea). Adicciones 2004; 16 (Supl 2): 75-82. Consultado octubre 2012. Disponible en: http://www. bvsde.paho.org/bvsacd/cd66/AgustinMontes.pdf

3. Ponciano-Rodríguez G, Morales-Ruiz A.Evaluación del Programa de Tratamiento para la Cesación del Tabaquismo en la clínica de la Facultad de Medicina de la UNAM. (En línea). Salud PúblicaMéx 2007; Vol. 49(sup 2):247-265. [Consultado noviembre 2012]. Disponible en: http://bvs.insp.mx/rsp/ articulos/articulo.php?id $=002000$

4. Comisión Nacional Antidrogas.Segundo estudio nacional sobre consumo de sustancias psicoactivas en población escolar de El Salvador SIDUC 2008. San Salvador, El Salvador. CNA/MINED/DIGESTYC/ MINEC. Informe final.

5. Bautista-Pérez F. Consumo de drogas psicoactivas lícitas e ilícitas y su incidencia en los problemas educacionales, familiares, económicos y legales en estudiantes universitarios de pregrado. Colección Drogas $N^{\circ} 1$. San Salvador. Universidad Evangélica de El Salvador/Universidad Gerardo Barrios. Ed. UEES. 1911.

6. Bautista-Pérez F. Policonsumo simultáneo de drogas en estudiantes universitarios de pregrado del área de la salud de una universidad, San Salvador-El Salvador. Texto y Contexto Enfermagen, Florianápolis, 2012; Brasil, Nºspecial 21:56-62.

7. De Gracia J, Faivovich D, Falcon F, Díaz R, Yentzen G, Kunstmann S. Prevalencia de tabaquismo y actitud de cambio frente al hábito tabáquico en universitarios chilenos: Importancia de la formación médica. Rev Chil Salud Pública 2009; Vol 13 (2): 72-81.
8. Villalobos A, Rojas R, MC. Consumo de tabaco en México. Resultados de las Encuestas Nacionales de Salud 2000 y 2006. Salud Pública Méx 2007; Vol. 49(sup 2):147-154.

9. Vicerrectoría del Medio Universitario-Programa Universidad Saludable. Caracterización del consumo de cigarillo en la comunidad universitaria de la Pontificia Universidad Javeriana, 2008-2009. (En línea).Consultada 23 diciembre 2013. Disponible en: https://nww.google.com. $\mathrm{v} / \# \mathrm{q}=$ tabaquismo+en+estudiantes+universitarios.

10. Navarro de Sáez M, Espig H, Medina V. Consumo de tabaco en estudiantes universitarios de Nuevo ingreso en escuelas de la salud. (En línea). Revista de Salud Pública, (XIV) 1 :54-61, jun. 2010. Consultada el 23 de diciembre 203. Disponible en: www.saludpublica.fcm. unc.edu.ar/.../RSP10_1_09_art6_pp\%2054_61.p.

11. Nerín I, Crucelaegui A, Novella P, Ramón y Cajal $P_{1}$ Sobradiel N, Gericó R. Encuesta sobre tabaquismo en estudiantes universitarios en relación con la práctica de ejercicio físico. (En línea). Arch Bronconeumol 2004;40(1):5-9. Consultado 23 diciembre 2013. Disponible en: http://www. archbronconeumol.org/es/vol-40-num-01/ sumario/13002520/

12. Chelet-Martí M, Escriche-Saura A, GarcíaHernández J, Moreno-Bas P. Consumo de tabaco en población universitaria de Valencia. (En línea). Trastornos Adictivos. 2011;13(1):5-10. www.elsevier. es/ta. consultado 23 diciembre 2013. Disponible en: http://zl.elsevier.es/es/revista/trastornosadictivos-182/consumo-tabaco-poblacionuniversitaria-valencia-90002624-originales-2011.

13. Uribe Barreto A, Lizaraso Soto F, Yushimito Rubiños $L$, Puelles Valqui J, Ramos Ramírez M, Ramírez Chipana M. et al. Prevalencia del tabaquismo en estudiantes universitarios. (En línea). Diagnóstico, Volumen 40, 1: enero-febrero 2001. Consultado, 23 diciembre 2013. Disponible en: http://www.fihu-diagnostico.org.pe/ revista/numeros/2001/enefeb01/6-12.html\#4.

14. Cordini MF, Cohen DC, Santa Cruz VS, Cook VL Consumo de tabaco en estudiantes de medicina. (En línea). Revista de 
Posgrado de la Vla Cátedra de Medicina. № 169 - Mayo 2007. Consultado 23 de diciembre 2013. Disponible en: http://.med.unne.edu.ar/revista/revista169/2_169.pdf.

15. The Survey System. Sample Size Calculator. Creative Research System. (En línea). [Consultado octubre 2012]. 2012. Disponible en: http://www. surveysystem.com/sscalc.htm.
16. Observatorio Interamericano sobre Drogas. Primer estudio sobre consumo de drogas y de otras problemáticas en población estudiantil de cinco universidades de El Salvador. Informe final. Editor Comisión Nacional Antidrogas. CNA/CICAD/OEA. 2011. Guía breve de SPSS 14. Chicago IL. EE.UU. snt. 